Nearly half of participants (48.4\%) were sexually inactive during last one year and $76.4 \%$ stated decrease or complete loss of sexual desire following the diagnosis. Diminished sexual desires showed significant association with ageing. Among sexually active women $92.5 \%$ had one sexual partner for last one year and $69.4 \%$ of sexually active women used condom at last coitus. Significant low condom usage seen among women with detectable viral load. None were found positive for Gonorrhea and Trichomoniasis. Prevalence of infectious syphilis was $0.3 \%$.

Conclusion Approximately half of WLHIV reported being sexually inactive despite satisfactory treatment outcomes and existence of a long term relationship. Findings demonstrate high condom use at last coitus, whilst low condom usage among women with detectable viral load need evaluation.

\section{P206 DOES THE PERFORMANCE AND OPERATIONAL SUPERIORITY OF POINT OF CARE TEST MAKE IT THE INVESTIGATION OF CHOICE IN CONFIRMING SYPHILIS?}

S Nagia*, A Bhargava, P Verma, N Khunger. Vardhman Mahavir Medical College, Apex Regional Std Centre, Safdarjung Hospital, New Delhi, India.i, New Delhi-110029, India

\subsection{6/sextrans-2021-sti.295}

Background According to World Health Organization, 6 million cases of syphilis occur every year, and low-income countries bear a $90 \%$ burden of the disease. Serological tests for syphilis form the mainstay of diagnosis for syphilis. Unlike various specific Treponemal tests, Rapid Immunochromatographic Test, also called the point-of-care test (POCT) for syphilis, does not require expert training or equipment. We evaluated the performance of POCT against other specific Treponemal tests for confirming the diagnosis of syphilis.

Methods Retrospectively data was analyzed of samples from the year 2017 to 2020 at Apex Regional STD centre, Safdarjung Hospital, New Delhi, which were tested by all the three treponemal test for syphilis, namely Treponema Pallidum Hemagglutination (TPHA), fluorescent treponemal antibody absorption test (FTA-ABS) and POCT. Sensitivity, Specificity, Positive Predictive Value (PPV), Negative Predictive Value (NPV), Diagnostic Accuracy of POCT, and TPHA were evaluated against the gold standard FTA-ABS.

Result A total of 599 samples were evaluated, of which $61.76 \%$ were positive by FTABS. Upon analysis, the sensitivity of POCT was 91.08\% (95\%CI: 87.70\%-93.78\%) and TPHA was $91.89 \%$ (95\%CI: $88.63 \%-94.46 \%)$, specificity of POCT was $89.08 \%$ (95\%CI: $84.31 \%-92.81 \%)$ and TPHA was 87.34\% (95\%CI: 82.32\%-91.35\%), PPV of POCT was $93.09 \%$ (95\%CI: $89.97 \%-95.48 \%)$ and TPHA was $92.14 \%$ (95\%CI: 88.91\%-94.67\%), NPV of POCT was $86.08 \%$ (95\%CI: $81 \%$ 90.22\%) and TPHA was $86.96 \%$ (95\%CI: 81.91-91.02\%) and Diagnostic accuracy of POCT was $90.32 \%$ and TPHA was 90.15\%.

Conclusion Owing to its operational superiority and higher specificity, POCT can replace TPHA for confirming the diagnosis of syphilis. POCT is affordable, equipment-free, has room temperature storage, and yields results within 15 minutes, enabling same-day testing and treatment. It can be used in a limited resource setting, community setup, or even self-testing. Wider patient reach will help improve patient identification and treatment, thereby strengthening STI prevention and control programmes.

\section{P207 EVALUATION OF OXFORD NANOPORE MINION SEQUENCING TO PREDICT ANTIMICROBIAL RESISTANCE PROFILES IN CLINICAL N. GONORRHOEAE STRAINS}

${ }^{1} \mathrm{~T}$ De Block*, 'I De Baetselier, 'S Abdellati, 1,2J Laumen, 'S Manoharan-Basil, 1,3 C Kenyon, ${ }^{1} \mathrm{D}$ Van den Bossche. 'Department of Clinical Sciences, Institute of Tropical Medicine, Antwerp, Belgium; 'Laboratory of Medical Microbiology, Vaccine and Infectious disease Institute, University of Antwerp, Antwerp, Belgium; ${ }^{3}$ Division of Infectious Diseases and HIV Medicine, University of Cape Town, Cape Town, South Africa

\subsection{6/sextrans-2021-sti.296}

Background Antimicrobial resistance (AMR) in N. gonorrhoeae (NG) is a big concern for public health and has an impact on treatment efficiency. Whole genome sequencing (WGS) of clinical strains can be used to predict AMR profiles based on detection of known resistance-associated mutations (RAM) and AMR gene acquisition. While Illumina short read sequencing gives low error rates, third-generation sequencing with Oxford Nanopore Technologies (ONT) has the advantage of generating long reads in real-time. We aimed to evaluate the ability of ONT sequencing to predict AMR in NG.

Methods WGS was performed on one azithromycin resistant and four ceftriaxone resistant clinical NG strains using ONT (MinION R9.4 flowcell) and Illumina (MiSEQ) sequencing platforms. Assemblies were obtained from: Illumina data (by SPAdes), ONT data (by Flye) and a hybrid assembly of both ONT and Illumina data (by Unicycler). Moreover, we evaluated four different polishing strategies (based on rebaler, racon and/or medaka) after Flye. Completeness of the assemblies were visually checked with bandage. AMR profile prediction based on RAM detection and AMR gene acquisition were obtained by using pathogenwatch and compared with phenotypic antimicrobial susceptibility testing.

Results Hybrid and ONT assemblies showed a complete, closed chromosome, while Illumina assemblies were not able to resolve genome structures. Pathogenwatch could predict AMR profiles (except for CRO) correctly on Illumina and hybrid assemblies, and detected RAM were concordant in both assemblies. RAM detection on ONT assemblies was inconsistent among the different polishing strategies. Moreover, fewer RAMs were detected which resulted in missed resistance in the predicted AMR profiles.

Conclusion Here, we demonstrate that ONT sequencing reveals the genomic structure of NG isolates, but high error rates leads to it missing RAMs in the consensus assembly. Polishing strategies of MinION based assemblies can reduce the proportion of RAMs missed but remain inferior to Illumina based assemblies.

\section{P208 EXPLORING THE DATA-SHARING ECOSYSTEM IN HIV CARE: HEALTHCARE PROFESSIONALS' BELIEFS AND PRACTICES}

${ }^{1} \mathrm{~K}$ Lloyd*, ${ }^{1} \mathrm{~S}$ Tariq, ${ }^{2} \mathrm{~A}$ Durrant, ${ }^{2} \mathrm{C}$ Claisse, ${ }^{3} \mathrm{~L}$ Coventry, ${ }^{4} \mathrm{~B}$ Kasadha, ${ }^{3} \mathrm{E}$ Sillence, ${ }^{5} \mathrm{~S}$ Stumpf, 1J Gibbs. Institute For Global Health, University College London, London, UK; ${ }^{2}$ Open Lab, School of Computing, Newcastle University, Newcastle upon Tyne, UK; ${ }^{3}$ Department of Psychology, Northumbria University, Newcastle upon Tyne, UK; ${ }^{4}$ Terrence Higgins Trust, London, UK; ${ }^{5}$ Department of Computer Science, City, University of London, London, UK

\subsection{6/sextrans-2021-sti.297}

Background Understanding data-sharing in HIV care is timely given the shift to remote consultations during COVID-19. We describe the 'data-sharing ecosystem' in HIV care by analysing 
HIV healthcare professional (HCP) beliefs and practices around sharing diverse types of service user and clinic-generated data across multiple contexts.

Methods During February-October 2020, we conducted 14 semi-structured interviews with HCPs working in a large UK HIV outpatient service. Participants engaged in a card sorting task, sorting 33 data types routinely shared in HIV care into categories (comfortable/not comfortable/not sure) across three data-sharing contexts: (a) service users to HCPs in consultations; (b) HCPs to GPs; (c) HCPs to non-HIV HCPs. Data were analysed thematically.

Results Over half (57\%) of participants were female; 57\% were doctors. Participants had worked in HIV for 12 years on average.

HCPs were comfortable with a wide range of data being shared with them by service users. Across all sharing contexts, HCPs were uncomfortable with sharing of service user photographs, perceiving them as not routinely shared, unnecessary, and potentially risking inadvertent sharing of inappropriate content. HCPs were comfortable sharing data with GPs and other non-HIV HCPs in two broad categories: (a) demographic data (e.g. age) and (b) non-sensitive data related to general health (e.g. sleep). HCPs were less comfortable sharing sensitive information about HIV status, sexual health, behaviour and identity. Service user consent and relevance of data to sharing context were key determinants of data-sharing comfort.

Conclusion Understanding the context of data-sharing in HIV care is increasingly important given the shift to remote consultations and expectations for self-management. We demonstrate the complex interplay of data types, relationship dynamics, and contexts of care provision that shape the data-sharing ecosystem in HIV care. Developing guidance on the sharing of service user and clinic-generated data in HIV care must account for these complexities.

\section{P209 SWITCHING TO DORAVIRINE IN CART EXPERIENCED PATIENTS: EFFECTIVE, HIGHLY TOLERATED, AND COST SAVING. A RETROSPECTIVE COHORT STUDY}

${ }^{1} \mathrm{~V}$ Lanting*, ${ }^{1} \mathrm{P}$ Oosterhof, ${ }^{1} \mathrm{D}$ Ait Moha, ${ }^{1} \mathrm{G}$ Van den Berk, ${ }^{1,2} \mathrm{~K}$ Brinkman. ${ }^{1}$ OLVG hospital, Amsterdam, The Netherlands; ${ }^{2}$ University of Amsterdam, Amsterdam, The Netherlands

\subsection{6/sextrans-2021-sti.298}

Background Doravirine is a NNRTI with demonstrated effect as third agent in treatment naive and experienced HIV patients.

Aim To evaluate outcomes of cART experienced patients switching their therapy to DOR/TDF/3TC or doravirine based regimens, with focus on efficacy, tolerability and costs.

Methods Baseline characteristics like treatment history, and laboratory values were retrospectively collected with follow up data at 6 months. If patients stopped therapy, reasons were evaluated. Baseline and follow-up data were compared in patients that continued therapy using IBM SPSS. Potential cost savings compared to prior cART were estimated for the cohort that continued DOR/TDF/3TC for follow-up period and extrapolated to the average per year.

Results A total of 377 patients (91\% men) were included that completed outpatient clinic visit or/and laboratory data at 6 months: $97.1 \%$ switched to Doravirine/tenofovir/lamivudine (DOR/TDF/3TC) and $2.9 \%$ to other doravirine based regimens. Doravirine was prematurely stopped in 58/377 patients (15.4\%). Reason to stop was a patient-reported adverse event in $43 / 377$ patients $(11.4 \%)$; most frequently gastrointestinal (3.8\%) or insomnia/sleep disturbances $(3.4 \%)$. Other reasons were virologic failure $(0,8 \%)$ with only 1 case demonstrating resistance (V106VA, V108VI), decreased renal function $(0,8 \%)$, and ALAT grade $2(0,5 \%)$.

Within the group that continued doravirine grade 1 ALAT elevation was more frequently observed at 6 months compared to baseline $(14.2 \%$ vs. $7.9 \%(p=0.004), n=318)$; no grade $2 /$ $3 / 4$ increase was observed. A decrease in LDL-c was seen $(3.50( \pm 1.10) \quad \mathrm{mmol} / \mathrm{L}$ vs. $2.97( \pm 0.79) \quad \mathrm{mmol} / \mathrm{L} ; \mathrm{n}=42$, $\mathrm{p}<0.001)$. No change in creatinine levels was observed. The switch in 319 patients that continued therapy saved 33\% in costs with an average saving of $€ 3,130$ euros per patient/year $(€ 1$ million total).

Conclusion Doravirine is a suitable therapy for maintenance treatment and shows enormous savings. In addition, our data shows that it is also effective and well tolerated by $85 \%$ in our patient group.

\section{P211 CHANGES IN SEXUAL BEHAVIOR DURING COVID-19 AMONG MEN WHO HAVE SEX WITH MEN IN A SEXUAL NETWORK STUDY}

${ }^{1} \mathrm{M}$ Spahnie*, ${ }^{1} \mathrm{~A}$ Matthews, ${ }^{1} \mathrm{D}$ Kiss, ${ }^{1} \mathrm{~L}$ Jones, ${ }^{2} \mathrm{C}$ Copen, ${ }^{2} \mathrm{~J}$ Rushmore, ${ }^{2} \mathrm{~K}$ Bernstein, ${ }^{1} \mathrm{~J}$ Ricks, ${ }^{1} \mathrm{~W}$ Miller, ${ }^{3} \mathrm{~A}$ Norris Turner. ${ }^{1}$ College of Public Health, The Ohio State University, Columbus, USA; ${ }^{2}$ Division of STD Prevention, Centers for Disease Control and Prevention, Atlanta, USA; ${ }^{3}$ College of Medicine, The Ohio State University, Columbus, USA

\subsection{6/sextrans-2021-sti.299}

Background As part of an ongoing sexual network study, we assessed the impact of COVID-19 on sexual behavior of men who have sex with men (MSM).

Methods The Columbus, Ohio arm of the multi-site Network Epidemiology of Syphilis Transmission (NEST) study is following 241 MSM over two years. Participants attend quarterly visits with behavioral surveys and HIV/STI testing. In April 2020, we implemented an additional survey to measure the impact of COVID-19 on sexual behavior. Our analysis compared participants' reported sexual behavior during two periods: April-July 2020 (T1) and August-December 2020 (T2).

Results Of the 200 participants who completed at least one COVID-19 survey in either time period, 10 (5\%) self-reported a previous COVID-19 diagnosis. We compared behaviors reported by 174 respondents who submitted surveys in both time periods. During T1, about half $(46 \%)$ said they felt more anxious about sex since COVID-19; anxiety was similar during T2 (43\%). Many reported engaging in online sexual activities, though we saw no meaningful differences by time period for starting or increasing chatting on hookup apps ( $\mathrm{T} 1=30 \%, \mathrm{~T} 2=30 \%)$, sexting $(\mathrm{T} 1=30 \%, \mathrm{~T} 2=25 \%)$, or video chat sex $(\mathrm{T} 1=16 \%, \mathrm{~T} 2=14 \%)$. Watching pornography was reported more often in T1 than T2 (53\% vs. 42\%). Nearly a third of respondents $(30 \%)$ in T1 reported that they were not having sex; this decreased to $17 \%$ in $\mathrm{T} 2$. Compared to prepandemic behavior, in T1 a majority of participants (74\%) said they were less likely to have sex with a new partner, compared to $61 \%$ during T2.

Conclusion During COVID-19, nearly half of MSM reported feeling more anxious about sex, and many turned to online activities in lieu of in-person sex. However, when comparing 